\title{
QUASIHARMONIC FUNCTIONS ON THE POINCARE $N$-BALL
}

\author{
BY LEO SARIO AND CECILIA WANG ${ }^{1}$
}

Communicated by W. T. Martin, February 8, 1973

Endowing an abstract Riemann surface with a conformal metric does not affect the harmonicity or the Dirichlet integral of a function on it. A fortiori, the classes $O_{G}, O_{H P}, O_{H B}, O_{H D}$ of Riemannian 2-manifolds which do not carry Green's functions or harmonic functions which are positive, bounded, or Dirichlet finite, are invariant under varying conformal metrics. Here the harmonicity is defined by $\Delta u=(d \delta+\delta d) u=0$, with $d$ the exterior derivative, $\delta$ the coderivative. In sharp contrast with the harmonic functions, the quasiharmonic functions [8], i.e., solutions of $\Delta u=1$, on a Riemannian manifold are greatly affected by conformal metrics, and consequently so are the corresponding null classes $O_{Q P}, O_{Q B}$, and $O_{Q D}$. A deep and interesting problem is to determine this dependence.

We study the problem in a concrete setup which (a) permits explicit results and (b) yields applications to the general biharmonic classification theory of Riemannian manifolds. The present work is devoted to the former aspect. The latter aspect, an elaborate question in its own right, will be discussed in later studies (e.g. [1], [2], [10]-[16]). Among the phenomena that will be encountered we mention here the following striking result (Hada-Sario-Wang [1]): On the $N$-ball $|x|<1$ with the metric $d s=\left(1-r^{2}\right)|d x|$ there exist Dirichlet finite nonharmonic biharmonic functions if and only if $N \leqq 10$.

The Riemannian manifold we choose for our present study of quasiharmonic functions is the $N$-ball $B_{\alpha}^{N}$ with the generalized Poincare metric

$$
d s=\left(1-r^{2}\right)^{\alpha}|d x|
$$

$\alpha$ a constant. We obtain the following complete characterizations:

$$
\begin{gathered}
B_{\alpha}^{N} \in O_{G} \Leftrightarrow \alpha \geqq \frac{1}{N-2}, \\
B_{\alpha}^{N} \in O_{Q P} \Leftrightarrow \alpha \notin\left(-1, \frac{1}{N-2}\right),
\end{gathered}
$$

\footnotetext{
AMS (MOS) subject classifications (1970). Primary 31B30.

${ }^{1}$ This work was sponsored by the U.S. Army Research Office-Durham, Grant DA-ARO-D-31-124-71-G181, University of California, Los Angeles.
} 


$$
\begin{aligned}
& B_{\alpha}^{N} \in O_{Q P} \Leftrightarrow \alpha \notin\left(-1, \frac{1}{N-2}\right), \\
& B_{\alpha}^{N} \in O_{Q D} \Leftrightarrow \alpha \notin\left(-\frac{3}{N+2}, \frac{1}{N-2}\right) .
\end{aligned}
$$

The proofs will appear in [9].

\section{BIBLIOGRAPHY}

1. D. Hada, L. Sario and C. Wang, Dirichlet finite biharmonic functions on the Poincaré $N$-ball, J. Reine Angew. Math. (to appear).

2. - , N-manifolds carrying bounded but no Dirichlet finite harmonic functions, Nagoya Math. J. (to appear).

3. Y. K. Kwon, L. Sario and B. Walsh, Behavior of biharmonic functions on Wiener's and Royden's compactifications, Ann. Inst. Fourier (Grenoble) 21 (1971), 217-226.

4. M. Nakai and L. Sario, A parabolic Riemannian ball, Proc. Sympos. Pure Math., vol. 11, Amer. Math. Soc., Providence, R.I., 1968, pp. 341-349. MR 38 \# 2703.

5. - Quasiharmonic classification of Riemannian manifolds, Proc. Amer. Math. Soc. 31 (1972), 165-169. MR 44 \#4692.

6. - Existence of Dirichlet finite biharmonic functions, Ann. Acad. Sci. Fenn. (to appear).

7. __ Existence of bounded biharmonic functions, J. Reine Angew. Math. 259 (1973), $147-156$.

8. L. Sario, Biharmonic and quasiharmonic functions on Riemannian manifolds, Duplicated lecture notes 1969-1970, University of California, Los Angeles, Calif.

9. L. Sario and C. Wang, Quasiharmonic functions on the Poincaré N-ball, Rend. Mat. (to appear).

10. - Radial quasiharmonic functions, Pacific J. Math. (to appear).

11. - Negative quasiharmonic functions, Tôhoku Math. J. (to appear).

12. - Parabolicity and existence of bounded biharmonic functions, Comm. Math. Helv. 47 (1972), 341-347.

13. - Parabolicity and existence of Dirichlet finite biharmonic functions, J. London Math. Soc. (to appear).

14. - Existence of Dirichlet finite biharmonic functions on the Poincaré 3-ball, Pacific J. Math. (to appear).

15. - Positive harmonic functions and biharmonic degeneracy, Bull. Amer. Math. Soc. 79 (1973), 182-187.

16. - Harmonic and biharmonic degeneracy, Kōdai Math. Sem. Rep. (to appear).

Department of Mathematics, University of California, los Angeles, California 90024 